\title{
Qualidade da carcaça e rendimento de cortes suínos com o uso de ractopamina
}

\author{
Carcass quality and yield of pork cuts with the use of ractopamine
}

\author{
Daniela de Souza Martins ${ }^{\mathrm{I}}$ Mónica Alvarado Soares $^{\mathrm{I}}$ Juliana Steffens $^{\mathrm{I}}$
}

\section{RESUMO}

\begin{abstract}
O objetivo deste trabalho foi avaliar a influencia do uso do aditivo ractopamina sobre os rendimentos dos cortes e a qualidade da carne suina ( $\mathrm{pH}$, cor, marmoreio e capacidade de retenção de água). Para os testes, foram utilizados 25 suínos alimentados com 7,5ppm de ractopamina na dieta e 25 suinos sem utilização deste aditivo. Os cortes foram obtidos através do fracionamento da carcaça em partes, sendo elas: pernil, paleta, sobrepaleta, barriga com costela e carré. Para os testes de rendimento de cortes padrão exportação, foram utilizadas 30 carcaças, sendo 15 de cada tratamento. Os resultados dos cortes de pernil e paleta foram expressos pela relação ao peso do corte preparado no padrão comercial, com relação ao peso da parte inteira obtida no momento que antecede a desossa. A utilização de Ractopamina na dieta promoveu redução no rendimento de toucinho de paleta $(7,32 \%)$ e toucinho de carré $(11,85 \%)$ e aumento no rendimento de carré sem toucinho (3,9\%). Nos cortes padrão comercial (com a separação de ossos e gorduras), observou-se maior rendimento no pernil $(7,46 \%)$ e na paleta $(9,03 \%)$ com o uso do aditivo. Em relação à qualidade da carne, observou-se que o uso de ractopamina na dieta não teve influência sobre a cor e capacidade de retenção de água e teve influência sobre a redução do marmoreio da carne e pH final. Desta forma, a utilização de ractopamina na dieta suína tem influência sobre o rendimento dos cortes padrão comercial e redução da gordura na carcaça, não exercendo influência negativa sobre a qualidade da carne suina.
\end{abstract}

Palavras-chave: aditivos, perda de peso, propriedades da carne.

\section{ABSTRACT}

The objective of this study was to evaluate the use of the additive ractopamine on the cuts yield and pork meat quality ( $\mathrm{pH}$, color, marbling and water holding capacity). For testing it was used 25 porks fed with 7.5ppm of ractopamine $f$ and 25 porks without use of the additive. The cuts were obtained by fractionation of carcass in parts, which are: ham palette, upper palette, belly with rib and loin. To yield of standard export cuts it was used 30 carcasses, 15 of each treatment. The results of ham cuts and palette were expressed by the weight of the cut prepared in commercial standard, in relation of the weight of the entire piece obtained at the time before boning. The use of ractopamine in diet induced a reduction in yield of bacon palette (7.32\%) and bacon loin (11.85\%) and an increase in the yield of loin without fat $(3.9 \%)$. In standard commercial cuts (with separation of bone and fat) it was observed higher yield shank $(7.46 \%)$ and palette $(9.03 \%)$ with the use of the additive. Regarding the quality of the meat, it was observed that the use of ractopamine in the diet had no influence on the color and water retention capacity and had influence on the reduction of marbling and the final pH. Thus, the use of ractopamine in pork diet influences the performance of commercial standard cuts and reduced carcass fat, not exerting negative influence on the quality of pork.

Key words: additives, weight loss, meat properties.

\section{INTRODUÇÃO}

Estudos e investimentos em suinocultura posicionaram o Brasil em quarto lugar no ranking de produção e exportação mundial de carne suína (ABIPECS, 2012; FAO, 2013). O manejo préabate e o aprimoramento gerencial dos produtores contribuíram para aumentar a oferta interna e colocar o País em destaque no cenário mundial (MAPA, 2012). Os principais cortes comercializados no mercado externo são pernil, paleta, lombo, sobrepaleta e miúdos suínos e os principais destinos da carne suína brasileira em 2012 foram Ucrânia (23,85\%), Rússia $(21,85 \%)$ e Hong Kong (21,45\%), representando, juntos, $67,15 \%$ do total exportado.

\footnotetext{
'Departamento de Pós-graduação em Engenharia de Alimentos, Universidade Regional Integrada do Alto Uruguai e das Missões (URI), Av. Sete de Setembro, 1621, 99700-000, Erechim, RS, Brasil. E-mail: julisteffens@uricer.edu.br. *Autor para correspondência. 
O mercado consumidor de alimentos, a nível mundial, está cada vez mais preocupado com os aspectos relacionados à saúde, sendo que a carne gorda aparece, em muitos casos, como sinônimo de baixa qualidade. Especialistas brasileiros investiram na evolução genética de suínos por 20 anos, o que reduziu em $31 \%$ a gordura da carne, $10 \%$ do colesterol e $14 \%$ de calorias, tornando a carne suína brasileira mais magra e nutritiva, além de saborosa (MAPA, 2012). Outra maneira de reduzir o conteúdo de gordura e promover o incremento de carne na carcaça é a utilização de aditivos alimentares na dieta nutricional dos animais. A ractopamina foi aprovada para uso no Brasil em 1996 e desde então seu efeito vem sendo amplamente estudado e seu uso disseminado para melhorar a qualidade da carne suína, especificamente a redução do conteúdo lipídico da carcaça (SANCHES, 2009).

Estudos apontam que animais tratados com ractopamina apresentam melhora em torno de $12 \%$ na conversão alimentar, o que proporciona também aumentonoganhodepesodiário, bem comoaumentono peso final (MARINHO et al., 2007; SANCHES, 2009).

Ucrânia, Hong Kong e outros 25 países como Uruguai, Argentina, Cingapura, Chile, Japão, Estados Unidos, Albânia, Venezuela, Angola, entre outros, permitem o uso de ractopamina na dieta animal, o que está sendo praticado no Brasil, porém a Rússia, Bloco Europeu e a China são extremamente exigentes quanto à garantia de ausência do uso de aditivos repartidores de energia na dieta animal (ABCS, 2013).

Nesse sentido, este trabalho teve por objetivo estudar a influência do uso de Ractopamina na dieta animal sobre a qualidade da carne da carcaça suína e sobre o rendimento dos cortes.

\section{MATERIAL E MÉTODOS}

Os experimentos foram conduzidos em um frigorífico abatedouro de suínos, situado em Santa Catarina, sendo utilizados suínos machos castrados, mestiços das raças Large White, Landrace e Agroceres PIC 337, tratados com diferentes tipos de ração na fase de terminação, sendo chamado de tratamento 1 para os suínos que receberam Ractopamina na dieta, onde a composição da ração era de $96,04 \%$ de proteína, $1,40 \%$ de gordura, $2,20 \%$ de aditivo, $7,5 \mathrm{ppm}$ de ractopamina e $0,36 \%$ de sal e tratamento 2 para os suínos que não receberam Ractopamina, no qual a composição da ração era de $97,54 \%$ de proteína, $0,60 \%$ de gordura, $1,50 \%$ de aditivo e $0,36 \%$ de sal. Todos foram criados em confinamento por 25 dias, com pocilgas cobertas e água à vontade. Os animais foram transportados conforme normas de bem estar animal. Após a descarga, os animais foram mantidos sob dieta hídrica por 6 horas, tempo após o qual foram conduzidos à linha de abate e insensibilizados via corrente elétrica (equipamento marca Sulmaq, Eletronarcose com 3 pontos de insensibilização) por aproximadamente 5 segundos (400 Volts, 1,4 Amperes). Foram abatidos pelo corte da veia jugular, sendo mantidos na posição horizontal para a sangria e, logo após, suspensos pelas patas traseiras em nórea de pendura. As carcaças foram conduzidas posteriormente para as etapas de escaldagem, toalete e evisceração, serradas longitudinalmente em duas partes, tipificadas, resfriadas em câmara de equalização de carcaças, com temperatura de aproximadamente $2 \pm 2^{\circ} \mathrm{C}$ por 24 horas e, posteriormente, as carcaças foram conduzidas para a etapa de desossa.

A perda de peso durante o resfriamento foi expressa pela medida do rendimento de carcaça fria sobre carcaça quente. Utilizaram-se 50 carcaças ( 25 de cada tratamento) para os testes de rendimento de carcaça fria sobre carcaça quente, sendo obtidos os resultados pela pesagem da carcaça quente (PQ); no momento da tipificação e pesagem da carcaça fria (PF); no momento da desossa, 24 horas após o abate. Para obtenção do rendimento, foi utilizada a relação da carcaça fria sobre a carcaça quente $\left(\mathrm{R}_{\mathrm{PF} / \mathrm{PQ}}\right)$.

Os cortes dos dois tratamentos foram obtidos através do fracionamento da carcaça em pernil, paleta, sobrepaleta, barriga com costela e carré. Os resultados foram expressos pela relação de peso da parte inteira obtida no momento que antecede a desossa, 24 horas após o abate, pelo peso da carcaça quente (PQ) obtido no momento da tipificação (KUTZLER et al., 2011).

Nos testes de rendimento de cortes padrão exportação, foram utilizadas 30 carcaças, sendo 15 de cada tratamento. A preparação dos cortes foi realizada pela separação das porções de pele, gordura, músculo e ossos, até a obtenção do padrão do corte comercial de pernil e paleta. Os resultados foram expressos pela relação de peso do corte preparado no padrão comercial, com relação ao peso da parte inteira obtida no momento que antecede a desossa, sendo eles: paleta sem toucinho $\left(\mathrm{P}_{\text {PALS/TOUC }}\right)$ e pernil sem pele $\left(\mathrm{P}_{\text {ER S/PELE }}\right)$.

A qualidade da carcaça foi avaliada por meio de análises dos parâmetros de $\mathrm{pH}$, cor, marmoreio e capacidade de retenção de água. As medidas de $\mathrm{pH}$ foram realizadas em 1 e $24 \mathrm{~h}$ após o abate (BRIDI et al., 2003; HINSON et al., 2011), a partir de amostra de pernil (Semimembranosus), utilizando pHmetro com eletrodo de inserção $(\mathrm{pH}$ 
320/Ste-1, WTW). Foram realizadas 5 coletas e a análise realizada em triplicata para cada tratamento.

Após 24 horas de resfriamento da carcaça na câmara frigorífica entre 0 e $2^{\circ} \mathrm{C}$, foram coletadas cinco amostras de aproximadamente $1 \mathrm{~cm}$ de espessura do músculo lombo (Longissimus dorsi), a partir da articulação da última vértebra torácica com a primeira vértebra lombar, para a realização das avaliações de cor, marmoreio e capacidade de retenção de água (CRA, drip loss). As amostras para avaliação de cor e marmoreio permaneceram expostas ao ar por um período de aproximadamente 20 minutos, para permitir a oxigenação do músculo e com o auxílio de padrões fotográficos (NATIONAL PORK PRODUCERS COUNCIL, 1989).

Para a avaliação da capacidade de retenção de água, medida através da perda de água por gotejamento, foi utilizada a metodologia de BRIDI et al. (2003) e MOORE et al. (2012). A perda de água por gotejamento (Drip loss) foi expressa pela diferença entre o peso da amostra no final do processo pelo peso inicial.

Os resultados foram analisados estatisticamente por Análise de Variância (ANOVA) e as médias comparadas pelo teste de Tukey, com $95 \%$ de confiança, utilizando o software STATISTIC 8.0.

\section{RESULTADOS E DISCUSSÃO}

Na tabela 1, são mostrados os resultados de rendimento, em relação ao rendimento de carcaça fria sobre carcaça quente, obtido em cada tratamento, onde se pode observar que não houve diferença significativa entre o rendimento de carcaça fria sobre a carcaça quente entre os dois tratamentos.

CARR et al. (2009) e HINSON et al. (2011) estudaram o efeito da ractopamina na dieta de suínos sobre o desempenho, características de carcaça e qualidade da carne de suínos em terminação e observaram que suínos tratados com ractopamina obtiveram maior peso de abate e de carcaça (quente e fria), porém não observaram diferença na perda de peso durante o resfriamento (relação entre peso de carcaça fria sobre peso de carcaça quente) nos diferentes tratamentos, o que está em acordo com os dados obtidos no presente estudo.

Na tabela 1, mostra-se o efeito do uso da ractopamina sobre o rendimento dos cortes inteiros sobre carcaça quente. O rendimento das partes inteiras componentes da carcaça foi obtido através do fracionamento da carcaça nas partes bruta e descrito como $\mathrm{R}_{\mathrm{PER}}$ (Rendimento de Pernil), $\mathrm{R}_{\mathrm{PAL}}$ (Rendimento

Tabela 1 - Efeito do uso de Ractopamina sobre o rendimento de carcaça fria sobre carcaça quente, rendimento dos Cortes inteiros sobre carcaça quente e rendimento de cortes padrão exportação e gordura.

\begin{tabular}{|c|c|c|c|c|}
\hline \multirow{2}{*}{ Medida } & \multicolumn{4}{|c|}{ 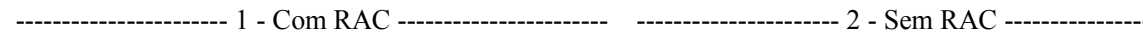 } \\
\hline & Média & Desvio Pad. & Média & Desvio Pad. \\
\hline \multicolumn{5}{|c|}{ } \\
\hline $\mathrm{PQ}(\mathrm{kg})$ & $86,82^{\mathrm{a}}$ & $\pm 7,09$ & $80,16^{\mathrm{b}}$ & $\pm 7,07$ \\
\hline $\mathrm{PF}(\mathrm{kg})$ & $85,16^{\mathrm{c}}$ & $\pm 6,71$ & $78,59^{\mathrm{d}}$ & $\pm 6,60$ \\
\hline $\mathrm{R}_{\mathrm{PF} / \mathrm{PQ}}$ & $98,10 \%{ }^{\mathrm{e}}$ & $\pm 0,62 \%$ & $98,08 \%{ }^{\mathrm{f}}$ & $\pm 0,89 \%$ \\
\hline \multicolumn{5}{|c|}{ - } \\
\hline $\mathrm{R}_{\mathrm{PER}}$ & $25,78^{\mathrm{a}}$ & $\pm 0,88$ & $25,81^{\mathrm{a}}$ & $\pm 1,12$ \\
\hline $\mathrm{R}_{\mathrm{PAL}}$ & $17,78^{\mathrm{b}}$ & $\pm 0,82$ & $17,96^{\mathrm{b}}$ & $\pm 0,67$ \\
\hline $\mathrm{R}_{\text {PAL S/TOUC }}$ & $14,95^{\mathrm{c}}$ & $\pm 0,92$ & $14,91^{\mathrm{c}}$ & $\pm 0,77$ \\
\hline $\mathrm{R}_{\mathrm{TOUC} . \mathrm{PAL}}$ & $2,83^{\mathrm{d}}$ & $\pm 0,47$ & $3,05^{\mathrm{e}}$ & $\pm 0,44$ \\
\hline $\mathrm{R}_{\text {SOBREP }}$ & $8,61^{\mathrm{f}}$ & $\pm 0,77$ & $8,82^{\mathrm{f}}$ & $\pm 0,52$ \\
\hline $\mathrm{R}_{\mathrm{BAR}}$ & $18,93^{\mathrm{g}}$ & $\pm 0,80$ & $18,50^{\mathrm{h}}$ & $\pm 0,74$ \\
\hline $\mathrm{R}_{\mathrm{CAR}}$ & $22,47^{\mathrm{i}}$ & $\pm 0,83$ & $22,15^{\mathrm{i}}$ & $\pm 1,02$ \\
\hline $\mathrm{R}_{\text {CAR S/ TOUC. }}$ & $19,45^{\mathrm{j}}$ & $\pm 0,78$ & $18,72^{\mathrm{k}}$ & $\pm 0,95$ \\
\hline \multicolumn{5}{|l|}{ Paleta } \\
\hline $\mathrm{R}_{\text {PALETA CORTE COMERCIAL }}$ & $57,02 \%{ }^{\mathrm{a}}$ & $\pm 3,92 \%$ & $52,29 \%^{\mathrm{b}}$ & $\pm 3,87 \%$ \\
\hline $\mathrm{R}_{\mathrm{GORDURA}}$ DA PALETA & $8,56 \%{ }^{\mathrm{c}}$ & $\pm 1,90 \%$ & $10,77 \%^{\mathrm{d}}$ & $\pm 3,54 \%$ \\
\hline \multicolumn{5}{|l|}{ Pernil } \\
\hline $\mathrm{R}_{\text {PERNIL CORTE COMERCIAL }}$ & $64,67 \%{ }^{\mathrm{e}}$ & $\pm 1,10 \%$ & $60,18 \%{ }^{\mathrm{f}}$ & $\pm 1,40 \%$ \\
\hline $\mathrm{R}_{\text {GORDURA DO PERNIL }}$ & $11,46 \%^{\mathrm{g}}$ & $\pm 1,53 \%$ & $12,24 \%^{\mathrm{g}}$ & $\pm 1,96 \%$ \\
\hline
\end{tabular}

*Letras iguais, na mesma linha, não diferem estatisticamente em nível de $5 \%$. 
de Paleta, subdividido em rendimento da paleta sem toucinho, $\mathrm{R}_{\mathrm{PALS} / \mathrm{TOUC}}$, e toucinho da paleta, $\mathrm{R}_{\text {TOUC. PAL }}$ ), $\mathrm{R}_{\mathrm{SP}}$ (Rendimento de Sobrepaleta), $\mathrm{R}_{\mathrm{BAR}}$ (Rendimento de Barriga com Costela), $\mathrm{R}_{\mathrm{CAR}}$ (Rendimento de Carré, subdividido em rendimento de Carré sem toucinho, $\mathrm{R}_{\text {CAR S/TOUC }}$, e toucinho do carré, $\mathrm{R}_{\text {TOUC. CAR }}$ ).

Conforme observado na tabela 1 , não há efeito significativo do uso de Ractopamina (7,5ppm) sobre o rendimento dos cortes inteiros de pernil, paleta inteira, paleta sem toucinho, sobrepaleta e carré inteiro, porém há redução no rendimento de toucinho da paleta $(\mathrm{P}<0,1)$ e toucinho do carré $(\mathrm{P}<0,05)$ no tratamento com ractopamina, em comparação ao tratamento sem o aditivo e aumento no rendimento de barriga com costela $(\mathrm{P}<0,05)$ e de carré sem toucinho $(\mathrm{P}<0,05)$ no tratamento com ractopamina, em comparação ao tratamento sem ractopamina, o que está de acordo com KUTZLER et al. (2011), quando suínos foram tratados com $6,2 \mathrm{ppm}$ de ractopamina.

$\mathrm{Na}$ avaliação do rendimento dos cortes padrão exportação, expressos como rendimento de pernil $\left(\mathrm{R}_{\text {PERNIL }}\right)$, rendimento de paleta $\left(\mathrm{R}_{\text {PALETA }}\right)$ e rendimento da gordura do pernil e da paleta $\left(\mathrm{R}_{\text {GORDURA }}\right)$, que podem ser observados na tabela 1 , obteve-se que o rendimento da paleta padrão comercial foi, em média, 9,03\% maior nas carcaças de suínos tratados com ractopamina, ao mesmo tempo em que o rendimento das gorduras foi, em média, 20,59\% menor neste mesmo tratamento, o que indica que a utilização do aditivo interfere sobre a quantidade de carne no corte de paleta. No entanto, CARR et al. (2009) observaram aumento de carne na paleta a partir do uso de 20ppm de ractopamina.

O rendimento do pernil padrão comercial foi $7,46 \%$ maior nas carcaças de suínos tratados com ractopamina, embora os rendimentos de gorduras não tenham obtido diferença significativa, o que pode ser atribuído ao aumento da espessura das fibras musculares. Resultados similares foram obtidos por BELLAVER et al. (1991), que observaram um aumento de $6 \%$ no rendimento de carne do pernil $(58,8 \%$ de rendimento no tratamento sem aditivo e $62,5 \%$ de rendimento no tratamento com o aditivo) e $15 \%$ de redução no rendimento de gordura do pernil (22,5\% de rendimento no tratamento sem aditivo e $19,1 \%$ de rendimento no tratamento com o aditivo) no tratamento com $10 \mathrm{ppm}$ de ractopamina frente ao tratamento sem o aditivo.

Na tabela 2, mostram-se os parâmetros de qualidade da carne, o que indicou não haver diferença na classificação da carne dos dois tratamentos, ficando ambos nos parâmetros de carne RFN (rosa avermelhado, firme e não exudativa). $\mathrm{O}$ uso de ractopamina não apresentou efeito significativo sobre
Tabela 2 - Efeito do uso de Ractopamina sobre as características de qualidade da carne: média, desvio padrão e nível de significância.

\begin{tabular}{lcccc}
\hline \multirow{2}{*}{ Medida } & \multicolumn{3}{c}{--- 1 - Com RAC ---- } & ---- 2 - Sem RAC ----- \\
& Média & Desvio Pad. & Média & Desvio Pad. \\
\hline pH inicial $^{1}$ & $6,38^{\mathrm{a}}$ & $\pm 0,23$ & $6,33^{\mathrm{a}}$ & $\pm 0,20$ \\
$\mathrm{pH} \mathrm{final}^{2}$ & $5,88^{\mathrm{b}}$ & $\pm 0,07$ & $5,61^{\mathrm{c}}$ & $\pm 0,08$ \\
Drip loss $(\%)$ & $4,59 \%^{\mathrm{d}}$ & $\pm 0,96 \%$ & $3,82^{\mathrm{d}}{ }^{\mathrm{d}}$ & $\pm 1,83 \%$ \\
Cor & $3,17^{\mathrm{e}}$ & $\pm 0,41$ & $3,33^{\mathrm{e}}$ & $\pm 0,52$ \\
Marmoreio & $2,00^{\mathrm{f}}$ & $\pm 0,00$ & $2,67^{\mathrm{g}}$ & $\pm 0,52$ \\
\hline
\end{tabular}

${ }^{1} \mathrm{pH}$ inicial medido $1 \mathrm{~h}$ após abate.

${ }^{2} \mathrm{pH}$ final $24 \mathrm{~h}$ após abate.

*Letras iguais, na mesma linha, não diferem estatisticamente ao nível de $5 \%$

o $\mathrm{pH}$ inicial, drip loss e cor, porém o tratamento com o aditivo apresentou maior $\mathrm{pH}$ final e menor quantidade de gordura marmorizada $(\mathrm{P}<0,05)$ do que o tratamento sem o uso ractopamina.

O pH mais elevado, observado no tratamento com Ractopamina, pode estar relacionado à redução das reservas de glicogênio do animal, devido à agitação e estresse dos suínos na propriedade em momento anterior ao abate (visto que o transporte de todos os animais foi realizado sob as mesmas normas), estando de acordo com os dados publicados por POLETTO et al. (2010), HINSON et al. (2011), quando foi constatado que suínos alimentados com ractopamina se tornam mais agressivos, o que pode ser a causa de estresse na propriedade.

$\mathrm{O}$ efeito observado do $\mathrm{pH}$ final elevado pode indicar aumento na tendência a se obter carne tipo DFD (escura, firme e seca), embora, na prática, esta tendência seja baixa e o efeito sobre a cor possa ser confundido com a concentração reduzida do pigmento heme nos músculos, por isso esta pode não aparecer mais escura (BRIDI \& SILVA, 2009). No presente estudo, não foi caracterizada carne DFD no tratamento com Ractopamina, pois cor e perda por gotejamento não foram característicos deste tipo de carne, e o $\mathrm{pH}$ final no tratamento com o aditivo, embora tenha valores superiores ao tratamento sem o aditivo, ainda estão abaixo de 6 , o que é característico de carne normal.

Segundo as análises realizadas para avaliar a qualidade da carne de carcaças com e sem a utilização de ractopamina na dieta dos suínos, foi possível observar que a ractopamina não tem influência sobre a classificação da qualidade da carne, pois os parâmetros observados nas análises de $\mathrm{pH}$, cor e drip loss classificaram ambas as carcaças no grupo de carne normal. O marmoreio que interfere na 
suculência da carne foi afetado, sendo que os animais que foram tratados com ractopamina apresentaram menor teor de gordura marmoreada, conforme análise realizada, o que indica que o uso de ractopamina diminui o conteúdo de gordura intramuscular da carne, tornando-a mais magra. AALHUS et al. (1990), estudando o efeito do uso de ractopamina (10ppm) na qualidade da carne, não observaram diferença nas atribuições de cor, marmoreio, $\mathrm{pH} 24$ horas e drip loss, quando comparado ao tratamento sem o aditivo.

A cor obtida para ambos os tratamentos realizados neste estudo encontra-se dentro da faixa considerada normal na carne suína, que, segundo BRIDI et al. (2003), é igual a três, que corresponde à cor rosa acinzentado (NPPC, 1989). HINSON et al. (2011) observou impacto significativo do uso de ractopamina $(7,4 \mathrm{ppm})(\mathrm{P}<0,05)$ sobre a cor, em que o tratamento apresentou escores menores para a cor (média 2,54 pontos em escala de 1 a 5) e o tratamento sem o aditivo apresentou média 2,89. Valores similares ao observado na avaliação do marmoreio do presente trabalho foram observados por GONZALEZ et al. (2010), quando ocorreu redução significativa no marmoreio das carcaças tratadas com ractopamina frente ao controle sem o aditivo.

Com relação à perda de água por gotejamento, assim como o observado no presente estudo, MOORE et al. (2012) e HINSON et al. (2011) não observaram diferença significativa na perda de água por gotejamento (drip loss) no tratamento com ractopamina frente ao controle.

Em relação ao rendimento de cortes suínos, foi possível observar, através dos resultados, que o rendimento de cortes é afetado pelo uso de ractopamina na dieta animal, pois o rendimento de pernil e paleta padrão comercial, toucinho da paleta, barriga com costela e carré sem toucinho foram influenciados significativamente, sendo que o rendimento de carne foi maior nos suínos tratados com ractopamina na dieta (observado pelo aumento de rendimento de pernil e paleta padrão comercial e de carré sem toucinho).

\section{CONCLUSÃO}

Os resultados deste trabalho demonstraram que o uso do aditivo tem influência sobre a deposição de proteína na fase de terminação, em detrimento da gordura, a ponto de afetar os volumes de carne e gordura produzidos na industrialização. Dessa forma, pode-se concluir que a utilização de ractopamina na dieta tem ação sobre o rendimento dos cortes padrão comercial e redução da gordura na carcaça, não exercendo influência negativa sobre a qualidade da carne suína.

\section{AGRADECIMENTOS}

Os autores agradecem a Universidade Regional Integrada do Alto Uruguai e das Missões (URI) pela infraestrutura.

\section{REFERÊNCIAS}

AALHUS, J.L. et al. The effect of ractopamine on performance, carcass composition and meat quality of finishing pigs. Canadian Journal of Animal Science, v.70, p.943-952, 1990.

ABCS (ASSOCIAÇÃO BRASILEIRA DOS CRIADORES DE SUÍNOS). Disponível em: <http://pubs.aic.ca/doi/abs/10.4141/ cjas90-113>. Acesso em: 08 set. 2013. doi: 10.4141/cjas90-113.

ABIPECS (ASSOCIAÇÃO BRASILEIRA DA INDÚSTRIA PRODUTORAE EXPORTADORADE CARNE SUÍNA). Disponível em: <http://www.abipecs.org.br>. Acesso em: 08 set. 2013.

BELLAVER, C. et al. Níveis de Ractopamina na dieta e efeitos sobre o desempenho e características de carcaça de suínos em terminação. Pesquisa Agropecuária Brasileira, v.26, n.10, p.1795-1802, 1991. Disponível em: <http://ainfo.cnptia.embrapa. br/digital/bitstream/AI-SEDE/21289/1/pab28_out_91.pdf >.

BRIDI A.M. et al. Efeito do genótipo halotano e de diferentes sistemas de produção na qualidade da carne suína. Revista Brasileira de Zootecnia, v.32, n.6, p.1362-1370, 2003. Disponível em: <http://www.scielo.br/pdf/rbz/v32n6/18425.pdf $>$. Acesso em: 20 março. 2013. doi: 10.1590/S1516-35982006000700021.

BRIDI, A.M.; SILVA, C.A. Avaliação da carne suína. Londrina: Midiograf, 2009. 120p.

CARR, S.N. et al. The effect of ractopamine hydrochloride (Paylean_) on lean carcass yields and pork quality characteristics of heavy pigs fed normal and amino acid fortified diets. Meat Science, v.81, p. 533-539, 2009. Disponível em: $<$ http://amena. $\mathrm{mx} / \mathrm{memorias} /$ ago2011/C05.pdf $>$. Acesso em: 10 abril. 2013. doi:10.1016/j.meatsci.2008.10.007.

FAO (FOOD AND AGRICULTURE ORGANIZATION OF THE UNITED NATIONS). Mapping supply and demand for animal-source foods to 2030. Disponível em: <http://www.fao. org/docrep/014/i2425e/i2425e00.htm>. Acesso em: 08 set. 2013.

GONZALEZ, J.M. et al. Effect of ractopamine- $\mathrm{HCl}$ supplementation for 28 days on carcass characteristics, muscle fiber morphometrics, and whole muscle yields of six distinct muscles of the loin and round. Meat Science, v.85, p.379-384, 2010. Disponível em: <http://www.sciencedirect.com/science/ article/pii/S0309174010000306>. Acesso em: 10 abril. 2013. doi: 10.1016/j.meatsci.2010.02.004.

HINSON, R.B. et al. Impact of dietary energy level and ractopamine on growth performance, carcass characteristics, and meat quality of finishing pigs. Journal of animal science, v.89, p.3572-3579, 2011. Disponível em: <http://www.ncbi.nlm.nih. gov/pubmed/21622875>. Acesso em: 1 abril. 2012. doi: 10.2527/ jas.2010-3302. 
KUTZLER, L.W. et al. Comparison of varying doses and durations of ractopamina hydrochloride on late-finishing pig carcass characteristics and meat quality. Journal of Animal Sciences, v.89, p.2176-2188, 2011. Disponível em: <http://www.ncbi.nlm. nih.gov/pubmed/21317349>. Acesso em: 03 março. 2012. doi: $10.2527 /$ jas.2010-3601

MAPA (MINISTÉRIO DA AGRICULTURA, PECUÁRIA E ABASTECIMENTO). Disponível em: $<\mathrm{http}: / /$ www.agricultura. gov.br/animal/especies/suinos>. Acesso em: 09 abr. 2012.

MARINHO, P.C. et al. Efeito da ractopamina e de métodos de formulação de dietas sobre o desempenho e as características de carcaça de suínos machos castrados em terminação. Revista Brasileira de Zootecnia, v.36, n.4, p.1061-1068, 2007. Disponível em: <http://www.scielo.br/pdf/rbz/v36n4s0/11.pdf>. Acesso em: 03 março. 2012. doi: 10.1590/S1516-35982007000500011.

MOORE, K.L. et al. The interaction between ractopamine supplementation, porcine somatotropin and moisture infusion on pork quality. Meat Science, v.92, p.125-131, 2012. Disponível em: < http:// www.sciencedirect.com/science/article/pii/S030917401200143X > Acesso em: 15 abril. 2012. doi: 10.1016/j.meatsci.2012.04.022.
NPPC (NATIONAL PORK PRODUCERS COUNCIL). Pork quality standards. National Pork Producers Council in cooperation with National Pork Board 4/99.04037. Des Moines, USA, 1989. 1p.

POLETTO, R. et al. Aggressiveness and brain amine concentration in dominant and subordinate finishing pigs fed the $\beta$-adrenoreceptor agonist ractopamine. Journal of Animal Science, v. 88, p. 3107 3120, 2010. Disponível em: <http://www.ncbi.nlm.nih.gov/ pubmed/20495130>. Acesso em: 2 março. 2013. doi: 10.2527/ jas.2009-1876.

SANCHES, J.F. Níveis de Ractopamina nas dietas de suínos machos castrados na fase de terminação. 2009. 56f. Dissertação (Mestrado em Ciência Animal) - Programa de Mestrado em Ciência Animal, Faculdade de Medicina Veterinária e Zootecnia, Universidade Federal do Mato Grosso do Sul, Campo Grande, MS.

USDA, U.S. Department of Agriculture, Agricultural Research Service. USDA National Nutrient Database for Standard Reference, Release 26. Nutrient Data Laboratory, 2013. Disponível em: <http://www.ars.usda.gov/ba/bhnrc/ndl>. Acesso em: 08 set. 2013. 